\title{
Article \\ Simulation Guided Hand-Driven Portable Triboelectric Nanogenerator: Design, Optimisation, and Evaluation
}

\author{
Yunzhong Wang, Anh Tran Tam Pham, Damian Tohl and Youhong Tang * $\mathbb{0}$ \\ Institute for Nanoscale Science and Technology, Medical Device Research Institute, \\ College of Science and Engineering, Flinders University, Tonsley 5042, Australia; \\ yunzhong.wang@flinders.edu.au (Y.W.); anh.pham@flinders.edu.au (A.T.T.P.); damian.tohl@flinders.edu.au (D.T.) \\ * Correspondence: youhong.tang@flinders.edu.au; Tel.: +61-8-82012138
}

check for updates

Citation: Wang, Y.; Pham, A.T.T.; Tohl, D.; Tang, Y. Simulation Guided Hand-Driven Portable Triboelectric Nanogenerator: Design,

Optimisation, and Evaluation.

Micromachines 2021, 12, 955.

https://doi.org/10.3390/

mi12080955

Academic Editors: Navneet Soin and Suzanne Smith

Received: 21 July 2021

Accepted: 6 August 2021

Published: 12 August 2021

Publisher's Note: MDPI stays neutral with regard to jurisdictional claims in published maps and institutional affiliations.

Copyright: (C) 2021 by the authors Licensee MDPI, Basel, Switzerland. This article is an open access article distributed under the terms and conditions of the Creative Commons Attribution (CC BY) license (https:/ / creativecommons.org/licenses/by/ $4.0 /)$.

\begin{abstract}
Inspired by the fundamental mechanics of an ancient whirligig (or buzzer toy; $3300 \mathrm{BC}$ ), a hand-driven rotational triboelectric nanogenerator (HDR-TENG) was designed and optimised, guided by our recently reported mathematical modelling. This modelling indicates that the power generated by HDR-TENG is a function of the number of segments, rotational speed, and tribosurface spacing with different weighting sensitivities. Based on the simulation results, additive manufacturing technology was combined with commercially available components to cost-effectively fabricate the HDR-TENG. The fabricated HDR-TENG can provide stable and adjustable rotational speed up to 15,000 rpm with a linear hand stretching. The output voltage of HDR-TENG maintains a constant value within 50,000 cycles of testing when using Nylon 66 and PTFE as the triboelectric material. It can charge a $47 \mu \mathrm{F}$ capacitor to $2.2 \mathrm{~V}$ in one minute. This study provides a cost-effective portable HDR-TENG device with adjustable high rotational speed, high power output, and long durable life, creating opportunities to provide a power supply for point-of-care devices in remote or resource-poor settings and applications in science and engineering education.
\end{abstract}

Keywords: hand driven; rotational triboelectric nanogenerator; simulation guidance; durability; capacitor charge

\section{Introduction}

The triboelectric nanogenerator (TENG) is one of the most popular methods to harvest electrical energy from mechanical energy such as vibration, sliding, rotation, etc. The main working modes of the triboelectric nanogenerator include the contact-separation mode and the sliding friction mode [1-4]. The contact-separation mode can significantly improve the durability life of the triboelectric material, but the output of the generator is relatively low compared to that of the sliding mode [5-8]. On the contrary, the sliding friction mode has greater output, but the triboelectric material's lifespan is very short [9-12]. To improve the TENG's workability, researchers have come to the strategy of the rotational TENG (R-TENG) [13-16]. This mechanism can not only provide adequate output but also lengthen the lifetime of the triboelectric material. Since the R-TENG operates in the grating-based sliding mode, its electrical output is advantageous for prolonged continuous energy harvesting.

R-TENG requires a starting force due to the friction and resistance forces that exist between the contact area of both triboelectric materials. For this reason, most existing R-TENG devices are driven by an AC/DC motor or some other external force such as wind energy or wave energy [17-21]. Using an AC/DC motor as the kinetic energy provider for R-TENG is a waste of energy because the AC/DC motor is driven by electricity. Alternatively, the forces provided by wind and ocean waves may not be enough to start R-TENG. Therefore, there exists the need for a rotational mode TENG, which provides adequate starting force and is highly portable. A recently reported hand-driven ultraspeed centrifuge easily operated by the linear reciprocating stretching and releasing of 
a wire attracted our attention [22,23]. This device has a low fabrication cost (20 cents), is lightweight $(2 \mathrm{~g})$, and can provide a maximum rotation speed of $12,800 \mathrm{rpm}$. It also provides a sufficient starting force while remaining portable and easy to operate. The combination of a manual hand-driven centrifuge and rotation TENG drives this research to innovate and apply hand-driven rational (HDR)-TENG [24]. We recently reported artificial intelligence enhanced mathematical modelling on R-TENG, considering the kinematic and geometric conditions [25] is used here to guide the HDR-TENG design. The optimal distributions for the charge, voltage, current, and harvested energy were all obtained from this simulation.

In this study, a high-speed HDR-TENG, optimised based on our mathematical modelling results, was fabricated, and evaluated with an easy operational method to convert human kinetic energy to electrical energy [26-30]. This HDR-TENG has significantly high durability, adjustable and stable rotation speeds, and the capability to charge a capacitor. The proposed HDR-TENG has applications for supplying power to point-of-care devices in remote or very remote or resource-poor settings and for science and engineering education.

\section{Experimental}

\subsection{Device Fabrication and Assembling}

These acrylic components in the HDR-TENG are cut by a laser cutter (Rayjet 300EDU, Australia), and the acrylic board has a thickness of $2 \mathrm{~mm}$. The rotary disk and the stationary disk have a $130 \mathrm{~mm}$ diameter according to the suggested mathematic modelling [25]. The ball-bearing stopper has an inner diameter of $22 \mathrm{~mm}$ and an outer diameter of $31 \mathrm{~mm}$. The other components are made by the 3D printing machine (Monoprice Maker Ultimate 2+, Rancho Cucamonga, CA, USA), and the material used is acrylonitrile butadiene styrene (ABS) plastic. The fasteners of the stationary and rotational parts have two holes with a diameter of $5 \mathrm{~mm}$, which are used to place the driven wire for rotating the device. Meanwhile, another two smaller holes with a diameter of $1.5 \mathrm{~mm}$ inside the fasteners are used to host cables, which transmit the electrical energy from the rotational part to the output of the HDR-TENG. In this device, the stainless-steel ball-bearing E2.6003-2Z/C3 (SKF, Australia) is selected for its durability and stainproof ability. The bearing's inner and outer diameters are $17 \mathrm{~mm}$ and $35 \mathrm{~mm}$, respectively. This ball-bearing has a $3.25 \mathrm{~N}$ static load when using the stock grease, and we change the grease to a low-friction type (6003-2RS/C3, Spain) to decrease the driving force of the ball bearing to ensure that the HDR-TENG can easily rotate during the operation.

\subsection{Triboelectric Material Selection}

The triboelectric materials selected in this study were Nylon 66 and polytetrafluoroethylene (PTFE) with a thickness of $250 \mu \mathrm{m}$, which belong in opposite spectra of the triboelectric series [31,32]. They were cut into fan shape pieces by a laser cutter with a circle radius of $45 \mathrm{~mm}$ (Rayjet 300EDU, Sydney, Australia). The materials were then cleaned with ethanol to remove the residues from the surfaces. Both triboelectric materials have a $200 \mathrm{~nm}$ gold coating (Q300T-D, Quorum, UK) on the contact surface and $50 \mathrm{~nm}$ chromium on the electrode surface. The surface charge density after coating of Nylon and PTFE are $1150 \mathrm{~kg} / \mathrm{m}^{3}$ and $2200 \mathrm{~kg} / \mathrm{m}^{3}$, respectively [25].

\subsection{Evaluation}

The full-bridge rectifier used to convert AC voltage (generated by the HDR-TENG) to $\mathrm{DC}$ voltage is shown in Figure 1. The $100 \mathrm{nF}$ capacitor is used to filter the high-frequency noise of the waveform to ensure that the waveform of DC voltage is as smooth as possible. The toggle switch is used to control the charging of the $47 \mu \mathrm{F}$ capacitor used for energy storage. 


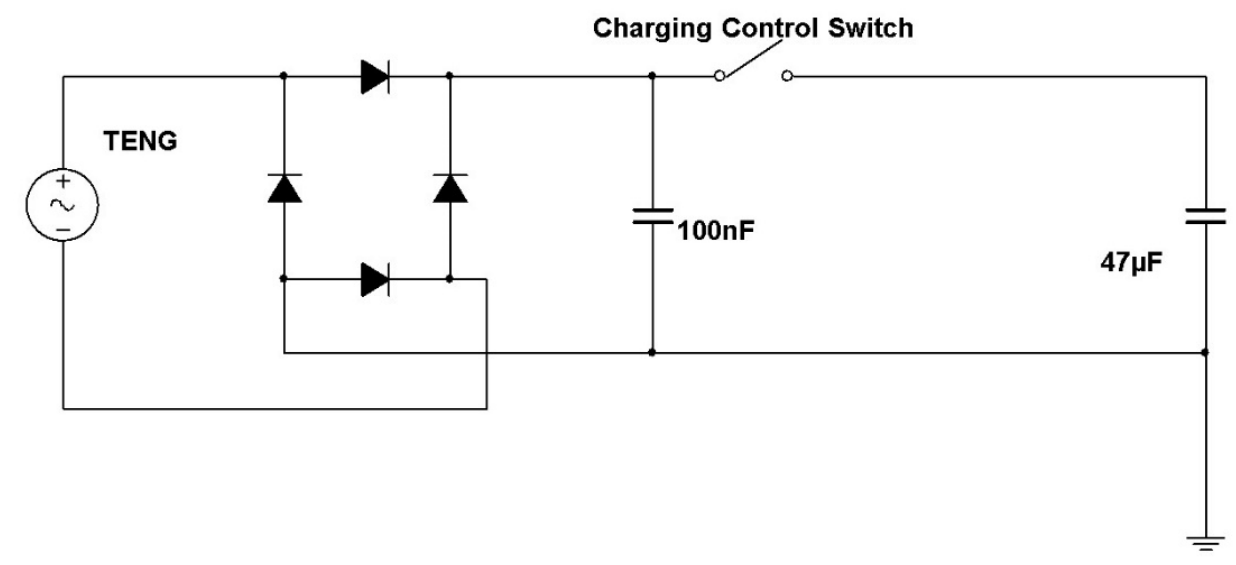

Figure 1. Circuit diagram of the full-bridge rectifier and charging circuit. The expected power supplier is the HDR-TENG device. The target charging component is the $47 \mu \mathrm{F}$ capacitor.

\section{Results and Discussion}

\subsection{Simulation Guided HDR-TENG Design}

The schematic view of the rotational TENG is shown in Figure 2 with the geometry parameters.

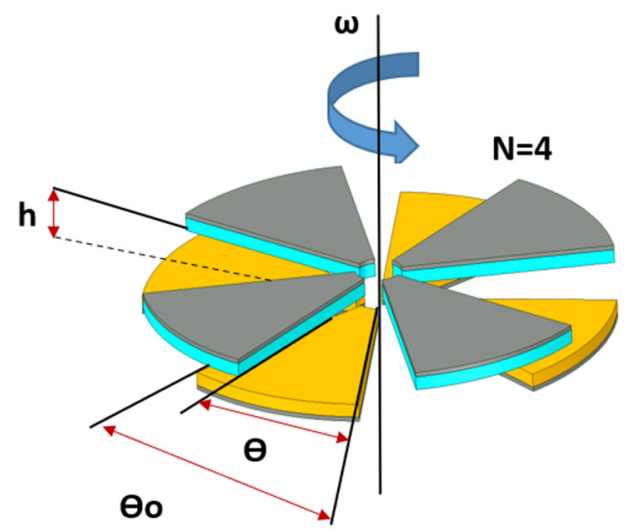

Figure 2. Schematic view of the rotational TENG, where $\theta_{0}$ and $\theta$ represent radians' sector and rotation angles, respectively. $N, h$, and $\omega$ are the grating number, the gap between triboelectric material, and the rotational speed.

From the previous simulation modelling [25], the short-circuit condition transferred charge $\left(Q_{S C}\right)$ is as follows:

$$
\begin{gathered}
Q_{S C}=\frac{N \sigma \theta\left(\frac{d_{1}}{\varepsilon_{1}}+\frac{d_{2}}{\varepsilon_{2}}\right)\left(r_{2}^{2}-r_{1}^{2}\right)}{2\left(\frac{d_{1}}{\varepsilon_{1}}+\frac{d_{2}}{\varepsilon_{2}}+h\right)}, \theta<\theta_{0} \\
Q_{S C}=\frac{N \sigma\left(2 \theta_{0}-\theta\right)\left(\frac{d_{1}}{\varepsilon_{1}}+\frac{d_{2}}{\varepsilon_{2}}\right)\left(r_{2}^{2}-r_{1}^{2}\right)}{2\left(\frac{d_{1}}{\varepsilon_{1}}+\frac{d_{2}}{\varepsilon_{2}}+h\right)}, \theta_{0}<\theta<2 \theta_{0}
\end{gathered}
$$

Short-circuit current $\left(I_{S C}\right)$ :

$$
\begin{gathered}
I_{S C}=\frac{d Q_{s c}}{d t}=\frac{N \sigma \frac{d \theta}{d t}\left(\frac{d_{1}}{\varepsilon_{1}}+\frac{d_{2}}{\varepsilon_{2}}\right)\left(r_{2}^{2}-r_{1}^{2}\right)}{2\left(\frac{d_{1}}{\varepsilon_{1}}+\frac{d_{2}}{\varepsilon_{2}}+h\right)}, \theta<\theta_{0} \\
I_{S C}=\frac{d Q_{s c}}{d t}=\frac{N \sigma \frac{d\left(2 \theta_{0}-\theta\right)}{d t}\left(\frac{d_{1}}{\varepsilon_{1}}+\frac{d_{2}}{\varepsilon_{2}}\right)\left(r_{2}^{2}-r_{1}^{2}\right)}{2\left(\frac{d_{1}}{\varepsilon_{1}}+\frac{d_{2}}{\varepsilon_{2}}+h\right)}, \theta_{0}<\theta<2 \theta_{0}
\end{gathered}
$$

where $\omega=\frac{d \theta}{d t}, d_{1}$ and $d_{2}$ are the thickness of dielectric 1 and 2, respectively, $\varepsilon_{1}$ and $\varepsilon_{2}$ are the relative permittivity of dielectric 1 and dielectric 2 , respectively. $\varepsilon_{0}$ is the vacuum 
permittivity, $\sigma$ is the charge density, and $r_{1}$ and $r_{2}$ are constants for the inner radius and outer radius of the TENG, respectively. Based on the relationship in Equation (2), the value of the short-circuit current is dependent on $h$, which is the gap between the two triboelectric materials and the rotation speed, $\omega$.

The capacitance, $C$, of the TENG can be calculated as follows:

$$
C=\frac{\varepsilon_{0} \frac{\left|\theta_{0}-\theta\right|\left(r_{2}^{2}-r_{1}^{2}\right)}{2}}{\frac{d_{1}}{\varepsilon_{1}}+\frac{d_{2}}{\varepsilon_{2}}+h}
$$

The expression between open-circuit voltage, $V_{o c}$, and the short-circuit condition transferred charge, $Q_{s c}$ is given as follows [14]:

$$
V_{O C}=\frac{Q_{S C}}{C}
$$

If we assume $C$ is a constant value and $Q_{s c}$ depends on $h$, which is the gap between the triboelectric material, as shown in Equation (2), the value of $V_{o c}$ will increase as $h$ decreases. The relationship between output voltage, $V$, and the charge, $Q$, is shown in Equation (5) [14], where $C$ is the capacitance of the TENG, and $Q$ is the total charge.

$$
V=R \frac{d Q}{d t}=-\frac{1}{C} Q+V_{O C}
$$

The main factor that affects the output voltage is the value of the load resistance, $R$, when $Q$ and $C$ are considered constants.

Our reported simulation modelling results [25] show that the resistance of the load is the major factor that will affect the output of the rotational mode TENG. Because the output power of the rotational mode TENG reaches the maximum value when the load resistance is in specific values. Other factors that will affect the output power are the gap between triboelectric material, the grating number, and rotational speed with decreased weightings. Based on the modelling results, a load resistance between $1 \mathrm{M} \Omega$ and $10 \mathrm{M} \Omega$ is the recommended range; a $10 \mathrm{M} \Omega$ load resistance has been selected for this study.

For the mechanism of R-TENG, the gap distance between two triboelectric materials, $h$, is shown in Figure 3a. The cycle of energy generation is shown in Figure 3b-d. When the rotational part starts to rotate, a flow of the electrons transfers from the negative charge material (PTFE) to the positive charge material (Nylon 66) to keep the electric neutrality between two triboelectric materials, as shown in Figure 3b. These two triboelectric materials need full separation to achieve the maximum charge, as shown in Figure 3c. A flow of electrons is then transferred from the positive charge material (Nylon 66) toward the negative charge material (PTFE) so that a current with the opposite direction can be generated, as shown in Figure 3d.

The HDR-TENG consists of eight components, as shown in Figure 3e. The first component is the copper brush holder used to hold the copper brush to collect the electrical energy from the Nylon 66. The L-shape support arm is used to position the device on a secured surface. The green ring is a limiting stopper used to hold the ball bearing. The grey disk is the stationary disk used to hold the PTFE. The groove on the stationary disk is designed to reduce the excess friction between two triboelectric materials, which will cause an unstable output waveform during the measurement. The pink and blue component is the high-speed low-friction ball bearing, which provides stable high-speed rotation and is an interconnection shaft between the stationary and rotational parts. The green disk represents the rotating disk used to hold the Nylon 66. The last component is the rotational core that is used to adjust the gap between two triboelectric materials. This component also assembles the rotational part and stationary part as one system. To operate the HDR-TENG, the rotational disk is first manually rotating to store the initial driving force for the HDR-TENG, then hand-to-linear reciprocating is used to stretch and release 
the wire, then the rotational part will be driven by force to generate electrical power, as shown in Figure 3f.

(a)

PTFE
Electrode

(c)

Separation of the top dielectric film

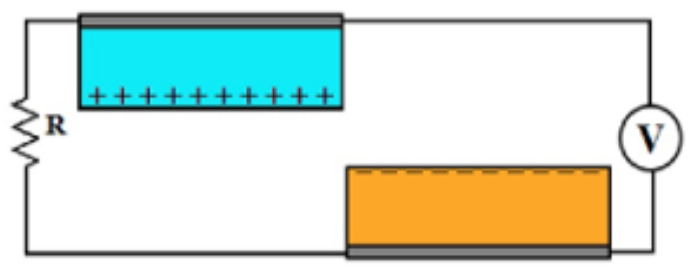

Full Separation of the dielectric layers (b)

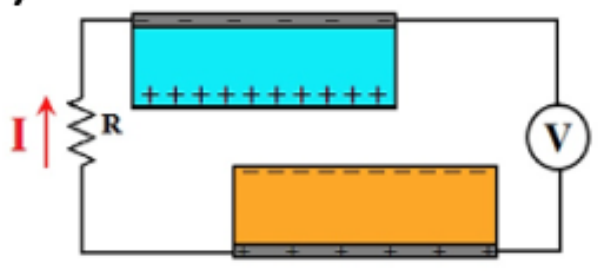

Rotation outwards of the top layer

(d)

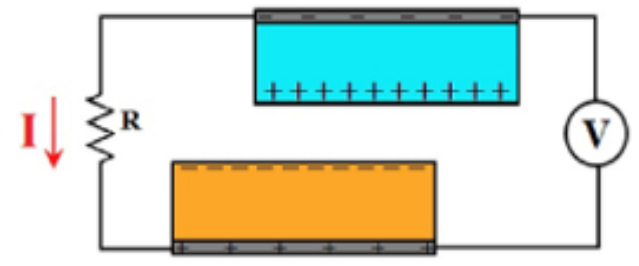

Rotation towards adjacent segment

(e)

Stationary part
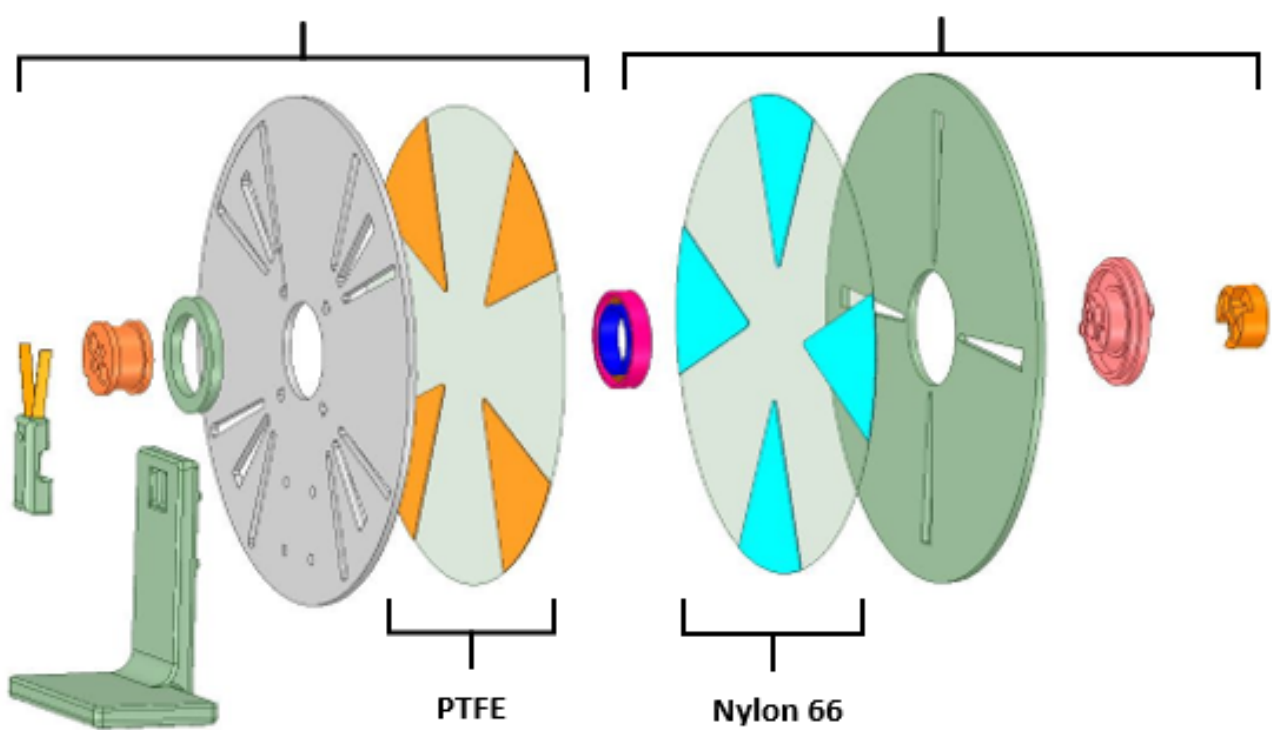

\section{Rotational part}
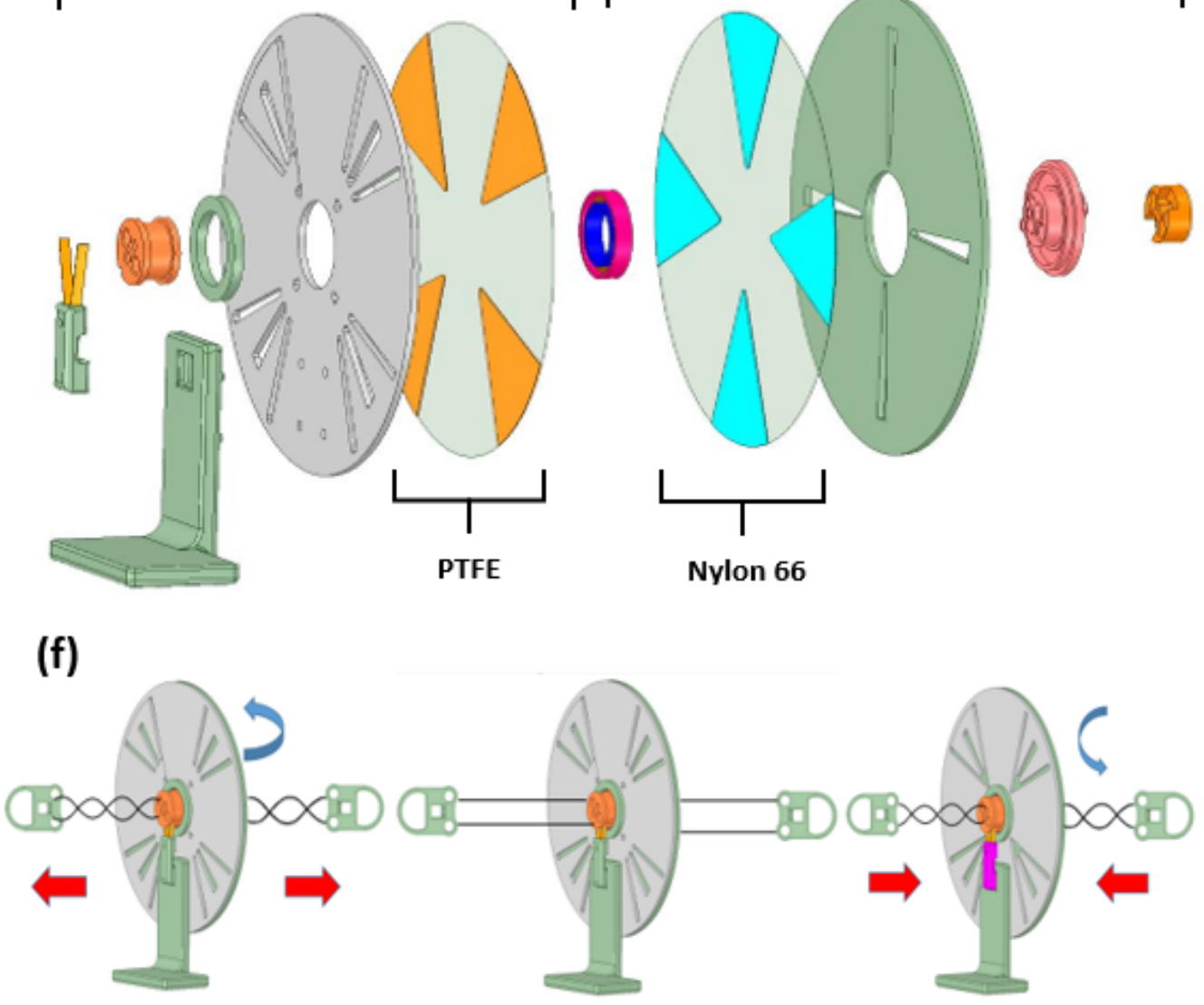

Figure 3. The working principle of the rotational TENG and the structure of the current HDR-TENG in this review; (a) two dielectric films are arranged so that there will be a controlled gap (h) between their surfaces; (b-d) the principle circuit applied on two dielectric films, When two dielectric films move relatively to each other, the change in the contact area generates the varying voltage in the circuit. Depending on the direction of the relative movements of two materials, the current in the circuit will have the corresponding direction; Reproduced with permission. [25]; (e) The explosion view of 
the HDR-TENG with all components divided into two groups of stationary part and rotational part. The orange and blue materials represent polytetrafluoroethylene (PTFE) and polyamide (Nylon 66). These two dielectric films, PTFE, and Nylon 66 are fixed on the two circle acrylic disks in grey and green. In the left-to-right order, the first component is the copper brush holder that is used to hold the copper brush (the orange V shape) that will collect the electrical energy from the Nylon 66; The L-shape support arm is used to position the whole device on a secured surface; The 2 orange and 1 pink pieces are used to hold two parts of the device together, as well as to collect the electrical energy from the rotational part (The green disk and the Nylon 66), then to transfer it to the copper brush component. The green ring is a limiting stopper used to hold the ball bearing. The grey disk with the PTFE dielectric film is the stationary part, which is fixed with the L-shape arm and will not rotate during the device' operation. The groove on the stationary disk is designed to reduce the excess friction between two triboelectric materials, which will cause an unstable output waveform during the measurement. The pink and blue component is the high-speed low-friction ball bearing. The green disk with the Nylon 66 is the rotational part, which will rotate during the device operation; ( $f$ ) the operation steps of the device; when stretching and releasing the wires, the rotating disk will accelerate from zero to its maximum rotation speed before decelerating to zero, then the rotating disk will reverse its rotation in the second period of stretching and releasing the wires.

\subsection{Device Optimisation}

Based on the mathematical modelling simulation result, the distance between two materials is directly proportional to the output power [25], which means the closer together the triboelectric materials are, the more electrical energy will be generated. However, a gap that is too small will cause the triboelectric materials to contact each other due to the tolerance of the 3D printed components, which will significantly reduce the useful life of the triboelectric material. Therefore, the open-circuit voltage, $V_{o c}$, of the HDR-TENG at the different gaps between stationary and rotational disks has been measured experimentally. When the gap between the two material films is larger than $1.5 \mathrm{~mm}$, the triboelectric materials do not have a triboelectric effect, resulting in the HDR-TENG failing to work at the optimum operating condition. When the gap is smaller than $1.2 \mathrm{~mm}$, the HDRTENG cannot rotate freely due to the excessive friction between triboelectric materials produced in the rotation. Therefore, gaps of $1.3 \mathrm{~mm}, 1.4 \mathrm{~mm}$, and $1.5 \mathrm{~mm}$ were chosen for further optimisation. The open-circuit voltage, $V_{o c}$, results of the HDR-TENG for these gaps are shown in Figure 4. The HDR-TENG can only generate $5 \mathrm{Vpp}$ voltage when the rotational and stationary elements gap is $1.5 \mathrm{~mm}$. The output of the HDR-TENG increases approximately four times to $18.9 \mathrm{Vpp}$ when the gap between the rotational element and stationary element is $1.4 \mathrm{~mm}$. When the gap is reduced to $1.3 \mathrm{~mm}$, the HDR-TENG can generate a voltage of $126.63 \mathrm{Vpp}$. Hence, the rotational core gap of $1.3 \mathrm{~mm}$ gap is the best choice for the HDR-TENG in this device.

The critical rotational speed, $\varphi_{\text {Critical }}$, is given as follows [22]:

$$
\varphi_{\text {Critical }}=\frac{L \sqrt{\pi^{2}-2^{2}}}{2 R_{S}}
$$

where $R_{S}$ is the string radius and $L$ is the string length.

The angular velocity is expressed as follows [22]:

$$
\omega=\sqrt{\frac{2 F_{m} R_{s}^{2}}{I L}}
$$

where, $F_{m}$ is the peak amplitude of the input force or the inertial moment of the rotation disc. 


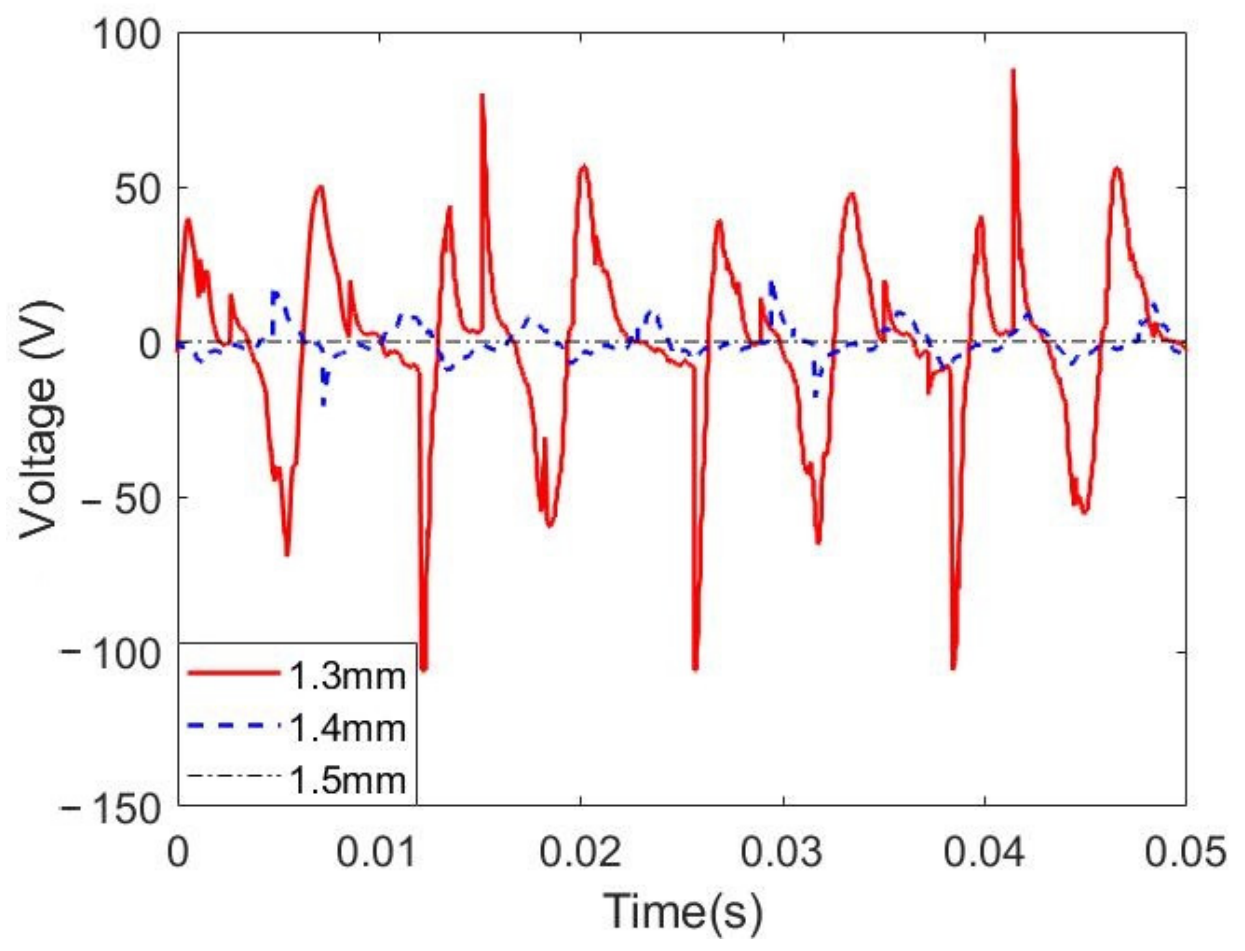

Figure 4. Optimization of the gap between rotational element and stationary element by measuring the open-circuit voltage, $V_{o c}$, of the HDR-TENG. Before reaching the contact point (the gap between two dielectric materials is $1.2 \mathrm{~mm}$ ), the output voltage of the device increases when the gap is reduced from $1.5 \mathrm{~mm}$ to $1.3 \mathrm{~mm}$ and reaches the maximum output voltage of $126.63 \mathrm{Vpp}$ with a gap of 1.3 $\mathrm{mm}$.

Then, the maximum rotational speed is as follows:

$$
\varnothing_{\text {Max }}=\varnothing_{\text {Critical }} \times \omega
$$

By substituting Equations (6) and (7) into Equation (8), the expression of the maximum rotational speed is obtained as follows:

$$
\varnothing_{\operatorname{Max}}=\frac{L \sqrt{\pi^{2}-2^{2}}}{2 R_{s}} \times \sqrt{\frac{2 F_{m} R_{s}^{2}}{I L}}=\sqrt{\frac{L F_{m}}{I}}
$$

As reported in the mathematical modelling [25], the rotational speed is directly proportional to the output power. In the HDR-TENG, the rotational speed can be controlled by the wire length when the input force and inertial moment are considered constants for the certain system, as given by Equation (9). Different wire lengths $(15 \mathrm{~cm}, 20 \mathrm{~cm}$, $25 \mathrm{~cm}, 30 \mathrm{~cm}, 35 \mathrm{~cm}$, and $40 \mathrm{~cm}$ ) were used to measure the HDR-TENG's open-circuit voltage, Voc, and short-circuit current results are shown in Figure 5. The open-circuit voltage of the HDR-TENG increases linearly with the length of wire, and the short-circuit current of the HDR-TENG starts to reach saturation when wire length is over $30 \mathrm{~cm}$. The saturation condition of the output current means most electrons existing on the surface of the triboelectric material are fully utilized by this optimised device [33]. The saturation condition has rarely shown up on the hand-driven mode TENGs before, with much lower rotating speeds [24]. Thus, with a stable high-rotation speed, the proposed device can successfully reach the saturation condition of the tested materials. 
(a)

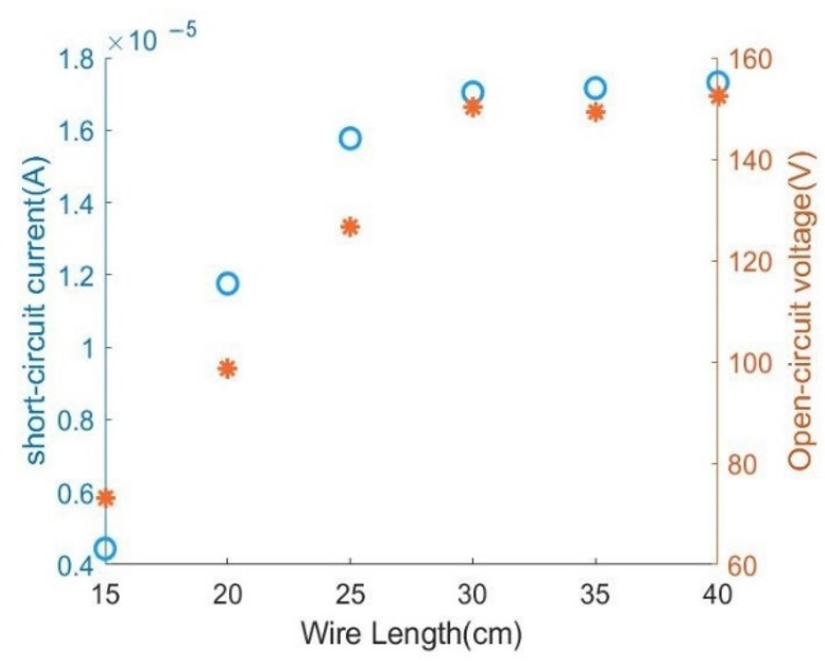

(b)

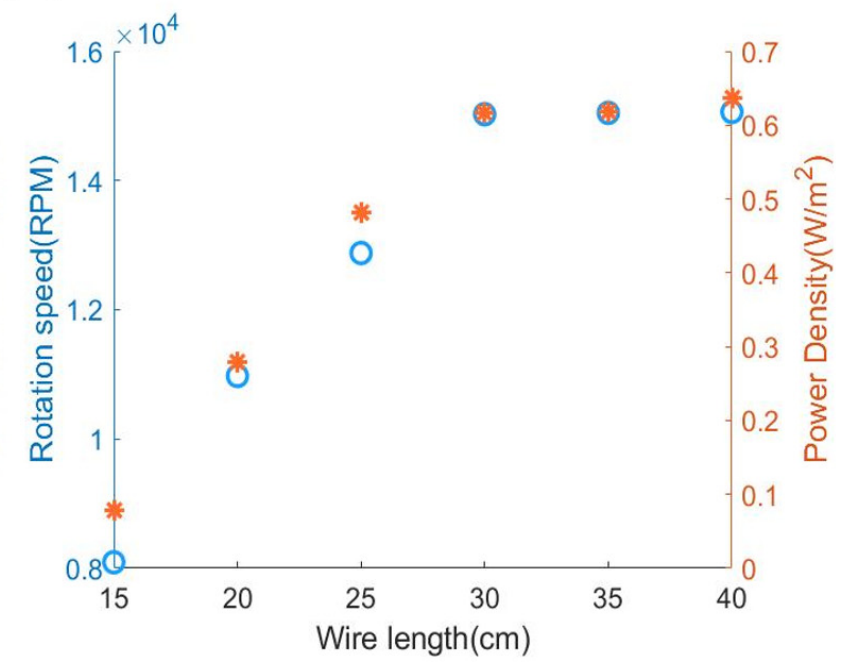

Figure 5. The result of HDR-TENG using different length cables. (a) Optimization of the wire length of the device. The blue circles represent the short-circuit current, and the open-circuit voltage is represented by the red stars. The load resistance for the test is $10 \mathrm{M} \Omega$. (b) Rotational speed and power density at different wire lengths.

From Figure 5a, it is clear the open-circuit voltage of HDR-TENG is directly proportional to the wire length. However, another factor requiring consideration in the design of the HDR-TENG is its ease of operation. Therefore, a wire length of $25 \mathrm{~cm}$ has been chosen to balance the voltage output and effective operation. Figure $5 \mathrm{~b}$ indicates the relationship between cable length and the output power of HDR-TENG. Based on these results, the optimum performance of the HDR-TENG was achieved with a gap of $1.3 \mathrm{~mm}$ between the stationary disk and the rotational disk is $1.3 \mathrm{~mm}$ and a wire length of $25 \mathrm{~cm}$, as in Figure $5 \mathrm{~b}$. These geometry and design parameters will be utilized in the HDR-TENG for its performance evaluation.

\subsection{Performance Evaluation}

The short-circuit current and the open-circuit voltage of the proposed HDR-TENG were measured with a maximum stabilised rotation speed of 12,800 rpm and compared with a $12 \mathrm{~V}$ DC motor-driven rotational mode TENG with a maximum stabilised rotation speed of $400 \mathrm{rpm}$ [25]. The geometry and triboelectric material setups for both the HDR-TENG and the DC motor-driven R-TENG are the same. From Equation (2), the output current of the rotational mode TENG is dependent on the rotation speed. The rotation speed of the DC motor reaches a maximum of $420 \mathrm{rpm}$ [25], while the proposed HDR-TENG device can achieve a speed of over $12,800 \mathrm{rpm}$, this dramatically improves the output of the TENG. The open-circuit voltage of the HDR-TENG is $126.63 \mathrm{Vpp}$, and the open-circuit voltage of the DC motor driven R-TENG is $32.95 \mathrm{Vpp}$, as shown in Figure 6a. The proposed HDR-TENG has an improvement in the output voltage of almost four times. The short-circuit current of the proposed HDR-TENG is $15.77 \mu \mathrm{A}$, while the DC motor-driven R-TENG can only produce $24.68 \mathrm{nA}$, as shown in Figure 6b. The proposed HDR-TENG shows an improvement in the short-circuit current of over 630 times. Based on this comparison, the hand-driven optimized design of the proposed HDR-TENG significantly shows improvement over the DC motor-driven R-TENG. 
(a)

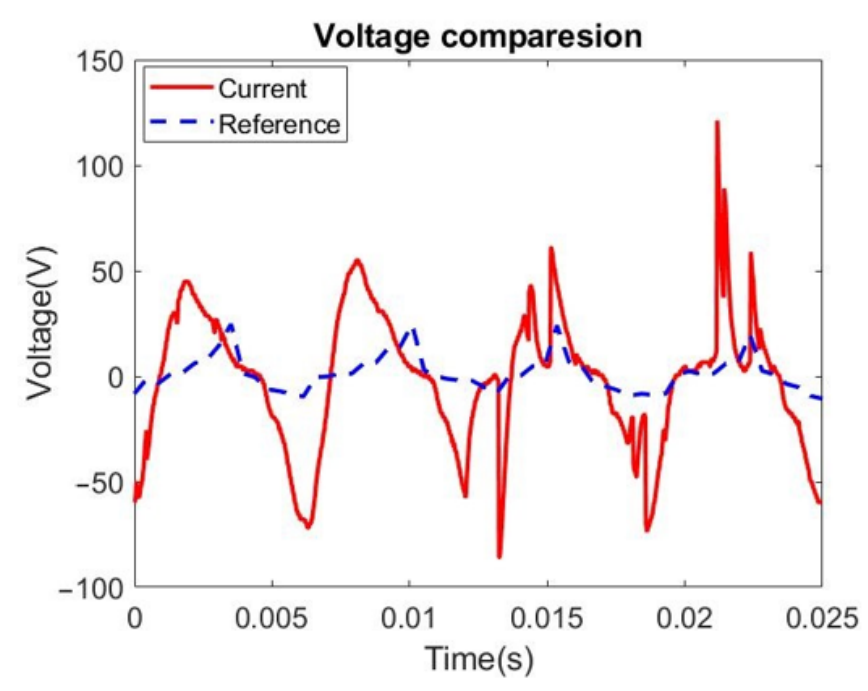

(b)

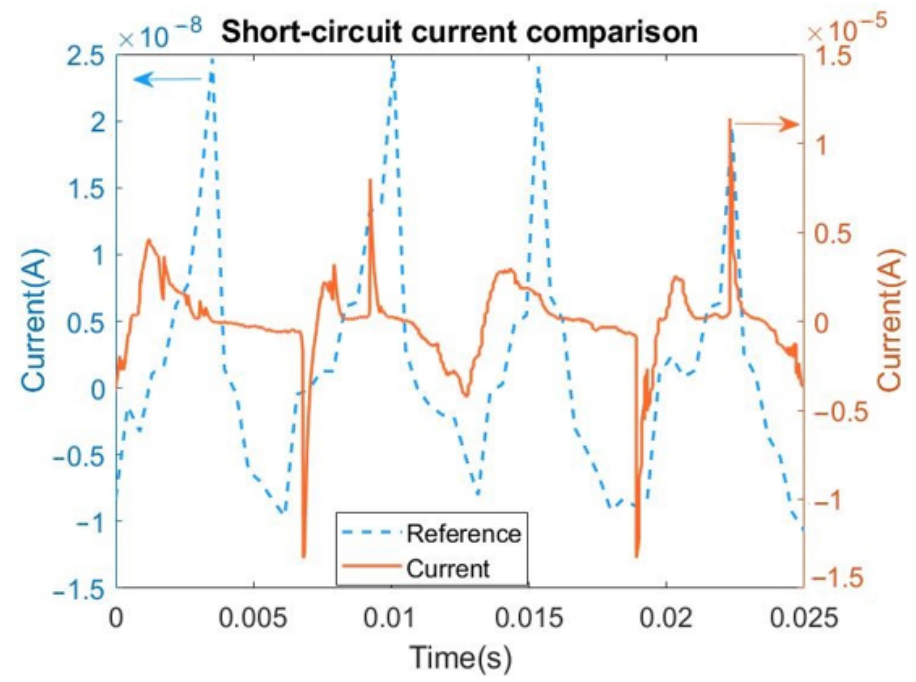

Figure 6. The results of the HDR-TENG and DC motor driven R-TENG for (a) the open-circuit voltage and (b) the shortcircuit current. The dashed blue line represents the results of the DC motor-driven R-TENG used in the previous study [25], and the solid red line represents the measurement results of the proposed HDR-TENG.

Figure 7 shows the power density of HDR-TENG under different loads. It can be seen that the power density increases with resistance until the load resistance reaches $10 \mathrm{M} \Omega$ and the power density is $0.481 \mathrm{~W} / \mathrm{m}^{2}$ in the experiment. The power density reduced significantly after when the load resistance increased from $10 \mathrm{M} \Omega$ to $1 \mathrm{G} \Omega$. Therefore, the output power of the HDR-TENG will reach its maximum when utilizing a $10 \mathrm{M} \Omega$ resistor as load.

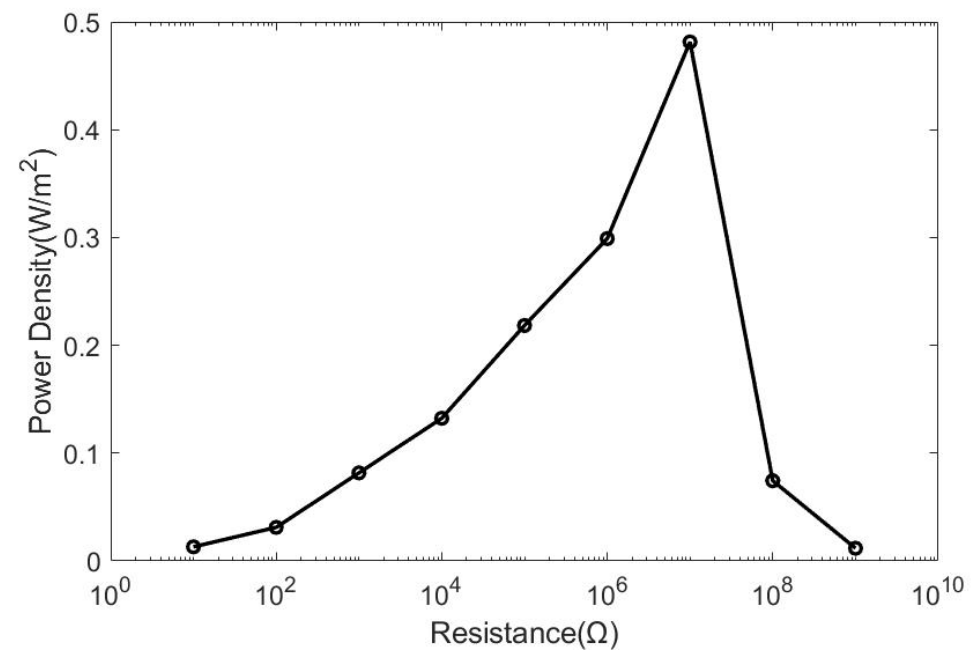

Figure 7. Power density of the HDR-TENG on the varying loading resistance. The specification of the HDR-TENG: $h$ is $1.3 \mathrm{~mm}$, the rotation speed is $12,800 \mathrm{rpm}$ with a $25 \mathrm{~cm}$ wire length, and the load resistance is $10 \mathrm{M} \Omega$.

The durability of the HDR-TENG has been evaluated, and the results are shown in Figure 8 . The solid red line represents the output voltage of a new HDR-TENG, which can generate $126.63 \mathrm{~V}$. The dashed blue line represents the output voltage of the HDR-TENG after 50,000 cycles of testing, which can generate $124.7 \mathrm{~V}$. The proposed optimization of the HDR-TENG can efficiently improve the useful life of the triboelectric material, which is one of the major existing problems for rotational mode TENG. 


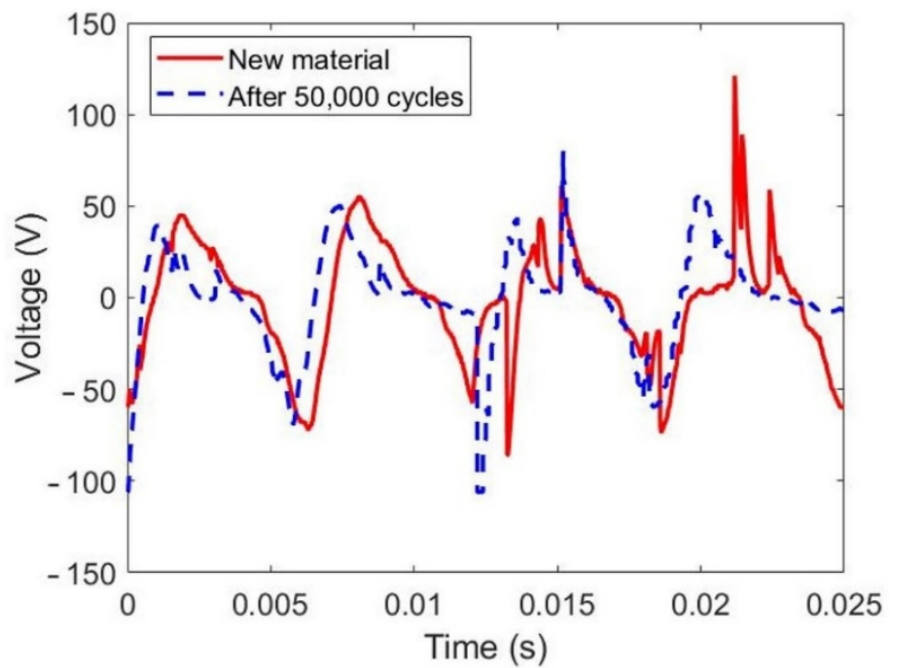

Figure 8. Durability evaluation of the output voltages of a new HDR-TENG compared to an HDRTENG after 50,000 cycles of rotation. The specification of the HDR-TENG: $h$ is $1.3 \mathrm{~mm}$, the rotation speed is $12,800 \mathrm{rpm}$ with a $25 \mathrm{~cm}$ wire length, and the load resistance is $10 \mathrm{M} \Omega$.

To verify the output of the HDR-TENG can be utilized, it was connected to the rectifier and charging circuit shown in Figure 9 and used to charge a capacitor. A digital multimeter was connected to the end of the $47 \mu \mathrm{F}$ capacitor to measure the charged voltage. Figure 9 shows that the $47 \mu \mathrm{F}$ capacitor reaches $2.2 \mathrm{~V}$ after $60 \mathrm{~s}$ of charging using the HDR-TENG to supply power.

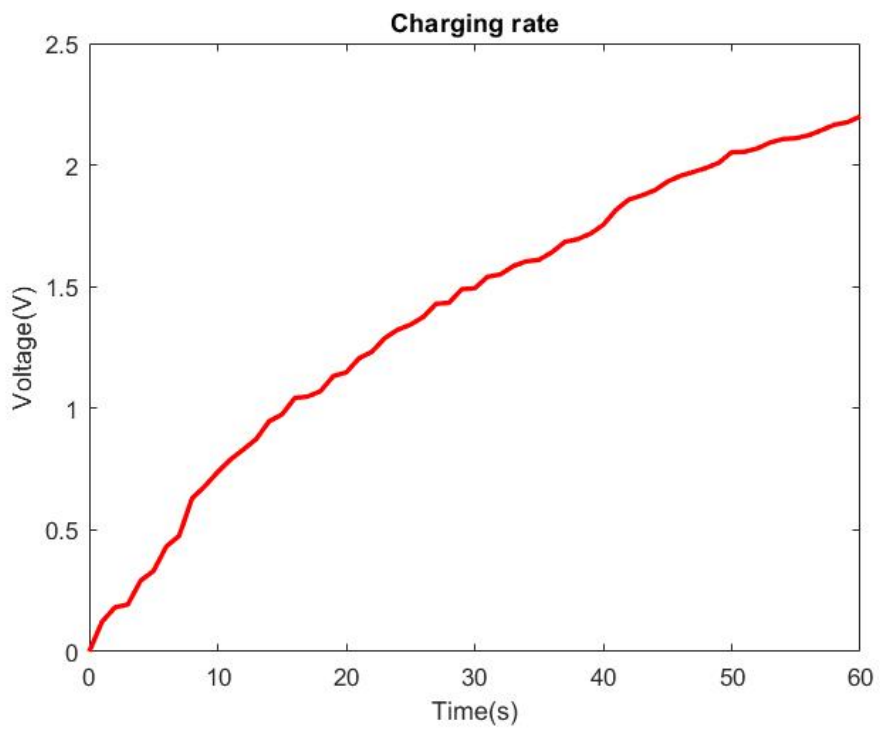

Figure 9. The charging curves of the $47 \mu \mathrm{F}$ capacitor with the HDR-TENG. The specification of the HDR-TENG: $h$ is $1.3 \mathrm{~mm}$, the rotation speed is $12,800 \mathrm{rpm}$ with a $25 \mathrm{~cm}$ wire length, and the load resistance is $10 \mathrm{M} \Omega$.

\section{Conclusions}

Guided by our previous reported simulation modelling, a cost-effective, controllable high-speed rotational hand-driven triboelectric nanogenerator has been proposed. The HDR-TENG enables continuous reciprocating stretching and releasing of a wire to obtain kinetic energy from the human body to actively control the performance of the rotational mode TENG. From our experimental results, the proposed HDR-TENG can utilize most electrons existing on the surface of the triboelectric material to generate current. The proposed HDR-TENG also works at an ultra-high rotational speed of over 12,800 rpm to 
provide a maximum output voltage of $126.63 \mathrm{~V}$ and a power density of $0.481 \mathrm{~W} / \mathrm{m}^{2}$. The power generated by our proposed HDR-TENG can sustainably charge a $47 \mu \mathrm{F}$ capacitor to $2.2 \mathrm{~V}$ in a minute and continues to have a stable output voltage after 50,000 cycles. The exchange charge density of the used triboelectric materials arising from the triboelectric charge exchange is $114.38 \mu \mathrm{C} / \mathrm{m}^{2}$. Based on the measurement result, the optimized device can efficiently utilize all the charges on the triboelectric material's surface to generate electrical energy. In the future study, we will search for the material with more charges on the surface to enhance the output power of the HDR-TENG.

Author Contributions: Conceptualization, Y.T.; Device design, A.T.T.P., Y.W.; Experiment, A.T.T.P., Y.W.; Data analysis, Y.W.; Writing, Y.W.; writing-review and editing, Y.T., D.T. All authors have read and agreed to the published version of the manuscript.

Funding: Yunzhong Wang is grateful to the financial support from College of Science and Engineering, Flinders University through Research Student Maintenance grant.

Acknowledgments: The authors acknowledge the expertise, equipment, and support provided by Microscopy Australia and the Australian National Fabrication Facility at the South Australian nodes under the National Collaborative Research Infrastructure Strategy and the Engineering Services Team in the College of Science and Engineering, Flinders University for their great support. The authors also thank for the initial discussion and simulation advice from Mohammad Khorsand in the group.

Conflicts of Interest: The authors declare that they have no known competing financial interests or personal relationships that could have appeared to influence the work reported in this publication.

\section{References}

1. Zhu, G.; Peng, B.; Chen, J.; Jing, Q.; Wang, Z.L. Triboelectric nanogenerators as a new energy technology: From fundamentals, devices, to applications. Nano Energy 2015, 14, 126-138. [CrossRef]

2. Wang, Z.L.; Jiang, T.; Xu, L. Toward the blue energy dream by triboelectric nanogenerator networks. Nano Energy 2017, 39, 9-23. [CrossRef]

3. Liu, W.; Wang, Z.; Wang, G.; Liu, G.; Chen, J.; Pu, X.; Xi, Y.; Wang, X.; Guo, H.; Hu, C.; et al. Integrated charge excitation triboelectric nanogenerator. Nat. Commun. 2019, 10, 1426. [CrossRef] [PubMed]

4. Wang, Z.L. On the first principle theory of nanogenerators from Maxwell's equations. Nano Energy 2020, 68, 104272. [CrossRef]

5. Yang, W.; Wang, X.; Li, H.; Wu, J.; Hu, Y. Comprehensive contact analysis for vertical-contact-mode triboelectric nanogenerators with micro-/nano-textured surfaces. Nano Energy 2018, 51, 241-249. [CrossRef]

6. Dharmasena, R.D.I.G.; Cronin, H.M.; Dorey, R.A.; Silva, S.R.P. Direct current contact-mode triboelectric nanogenerators via systematic phase shifting. Nano Energy 2020, 75, 104887. [CrossRef]

7. Chen, H.; Zhang, J.; Wu, W.; Song, G. Theoretical system of contact-mode triboelectric nanogenerators for high energy conversion efficiency. Nanoscale Res. Lett. 2018, 13, 346. [CrossRef] [PubMed]

8. Xu, C.; Song, Y.; Han, M.; Zhang, H. Portable and wearable self-powered systems based on emerging energy harvesting technology. Microsyst. Nanoeng. 2021, 7, 25. [CrossRef]

9. Khorsand, M.; Tavakoli, J.; Kamanya, K.; Tang, Y. Simulation of high-output and lightweight sliding-mode triboelectric nanogenerators. Nano Energy 2019, 66, 104115. [CrossRef]

10. Shao, J.; Jiang, T.; Tang, W.; Xu, L.; Kim, T.M.; Wu, C.; Chen, X.; Chen, B.; Xiao, T.; Bai, Y.; et al. Studying about applied force and the output performance of sliding-mode triboelectric nanogenerators. Nano Energy 2018, 48, 292-300. [CrossRef]

11. Xia, K.; Du, C.; Zhu, Z.; Wang, R.; Zhang, H.; Xu, Z. Sliding-mode triboelectric nanogenerator based on paper and as a self-powered velocity and force sensor. Appl. Mater. Today 2018, 13, 190-197. [CrossRef]

12. Zhang, W.; Diao, D.; Sun, K.; Fan, X.; Wang, P. Study on friction-electrification coupling in sliding-mode triboelectric nanogenerator. Nano Energy 2018, 48, 456-463. [CrossRef]

13. Lin, Z.; Zhang, B.; Zou, H.; Wu, Z.; Guo, H.; Zhang, Y.; Yang, J.; Wang, Z.L. Rationally designed rotation triboelectric nanogenerators with much extended lifetime and durability. Nano Energy 2020, 68, 104378. [CrossRef]

14. Jiang, T.; Chen, X.; Yang, K.; Han, C.; Tang, W.; Wang, Z.L. Theoretical study on rotary-sliding disk triboelectric nanogenerators in contact and non-contact modes. Nano Res. 2016, 9, 1057-1070. [CrossRef]

15. Wang, Z.L. Triboelectric nanogenerators as new energy technology and self-powered sensors-Principles, problems and perspectives. Faraday Discuss. 2015, 176, 447-458. [CrossRef] [PubMed]

16. Zhao, C.; Zhang, Q.; Zhang, W.; Du, X.; Zhang, Y.; Gong, S.; Ren, K.; Sun, Q.; Wang, Z.L. Hybrid piezo/triboelectric nanogenerator for highly efficient and stable rotation energy harvesting. Nano Energy 2019, 57, 440-449. [CrossRef]

17. Chen, X.; Ma, X.C.; Ren, W.W.; Gao, L.X.; Lu, S.; Tong, D.Q.; Wang, F.Y.; Chen, Y.; Huang, Y.; He, H.; et al. A Triboelectric Nanogenerator Exploiting the Bernoulli Effect for Scavenging Wind Energy. Cell Rep. Phys. Sci. 2020, 1, 100207. [CrossRef] 
18. Cho, Y.; Lee, K.; Park, S.; Ahn, S.; Kim, W.; Kim, J.; Park, S.; Sun, J.; Jung, C.; Chung, J.; et al. Rotational wind power triboelectric nanogenerator using aerodynamic changes of friction area and the adsorption effect of hematoxylin onto feather based on a diversely evolved hyper-branched structure. Nano Energy 2019, 61, 370-380. [CrossRef]

19. Bai, P.; Zhu, G.; Liu, Y.; Chen, J.; Jing, Q.; Yang, W.; Ma, J.; Zhang, G.; Wang, Z.L. Cylindrical rotating triboelectric nanogenerator. ACS Nano 2013, 7, 6361-6366. [CrossRef]

20. Kim, T.; Chung, J.; Kim, D.Y.; Moon, J.H.; Lee, S.; Cho, M.; Lee, S.H.; Lee, S. Design and optimization of rotating triboelectric nanogenerator by water electrification and inertia. Nano Energy 2016, 27, 340-351. [CrossRef]

21. Yong, H.; Chung, J.; Choi, D.; Jung, D.; Cho, M.; Lee, S. Highly reliable wind-rolling triboelectric nanogenerator operating in a wide wind speed range. Sci. Rep. 2016, 6, 33977. [CrossRef] [PubMed]

22. Bhamla, M.S.; Benson, B.; Chai, C.; Katsikis, G.; Johri, A.; Prakash, M. Hand-powered ultralow-cost paper centrifuge. Nature Biomed. Eng. 2017, 1, 0009. [CrossRef]

23. Byagathvalli, G.; Pomerantz, A.; Sinha, S.; Standeven, J.; Bhamla, M.S. A 3D-printed hand-powered centrifuge for molecular biology. PLoS Biol. 2019, 17, e3000251. [CrossRef] [PubMed]

24. Zou, Y.; Xu, J.; Fang, Y.; Zhao, X.; Zhou, Y.; Chen, J. A hand-driven portable triboelectric nanogenerator using whirligig spinning dynamics. Nano Energy 2021, 83, 105845. [CrossRef]

25. Khorsand, M.; Tavakoli, J.; Guan, H.; Tang, Y. Artificial intelligence enhanced mathematical modeling on rotary triboelectric nanogenerators under various kinematic and geometric conditions. Nano Energy 2020, 75, 104993. [CrossRef]

26. Peng, X.; Dong, K.; Ye, C.; Jiang, Y.; Zhai, S.; Cheng, R.; Liu, D.; Gao, X.; Wang, J.; Wang, Z.L. A breathable, biodegradable, antibacterial, and self-powered electronic skin based on all-nanofiber triboelectric nanogenerators. Sci. Adv. 2020, 6, eaba9624. [CrossRef]

27. Cho, S.; Hanif, Z.; Yun, Y.; Khan, Z.A.; Jang, S.; Ra, Y.; Lin, Z.-H.; La, M.; Park, S.J.; Choi, D. Triboelectrification-driven microbial inactivation in a conductive cellulose filter for affordable, portable, and efficient water sterilization. Nano Energy 2021, 88, 106228. [CrossRef]

28. Dong, K.; Hu, Y.; Yang, J.; Kim, S.-W.; Hu, W.; Wang, Z.L. Smart textile triboelectric nanogenerators: Current status and perspectives. MRS Bull. 2021, 46, 512-521. [CrossRef]

29. Dong, K.; Peng, X.; An, J.; Wang, A.C.; Luo, J.; Sun, B.; Wang, J.; Wang, Z.L. Shape adaptable and highly resilient 3D braided triboelectric nanogenerators as e-textiles for power and sensing. Nat. Commun. 2020, 11, 2868. [CrossRef]

30. Yun, Y.; Jang, S.; Cho, S.; Lee, S.H.; Hwang, H.J.; Choi, D. Exo-shoe triboelectric nanogenerator: Toward high-performance wearable biomechanical energy harvester. Nano Energy 2021, 80, 105525. [CrossRef]

31. Zou, H.; Zhang, Y.; Guo, L.; Wang, P.; He, X.; Dai, G.; Zheng, H.; Chen, C.; Wang, A.C.; Xu, C.; et al. Quantifying the triboelectric series. Nat. Commun. 2019, 10, 1427. [CrossRef] [PubMed]

32. Henniker, J. Triboelectricity in polymers. Nature 1962, 196, 474. [CrossRef]

33. Wu, C.S.; Wang, C.; Ding, W.B.; Guo, H.Y.; Wang, Z.L. Triboelectric nanogenerator: A foundation of the energy for the new era. Adv. Energy Mater. 2019, 9, 1802906. [CrossRef] 